\title{
AVALIAÇÃO DO NÍVEL DE CONHECIMENTO E QUALIDADE DA ATENÇÃO FARMACÊUTICA OFERTADA A USUÁRIOS DE MEDICAMENTOS EM SILVIANÓPOLIS, SUL DE MINAS GERAIS, BRASIL
}

\author{
Luana Jéssica de PAIVA ${ }^{1}$ \\ Luis Henrique Sales OLIVEIRA ${ }^{2}$ \\ Alexandre de SOUZA e SILVA ${ }^{3}$ \\ Valdomiro Vagner de SOUZA* ${ }^{4,5,6}$ \\ ${ }^{I}$ Farmacêutica. Faculdade de Ciências da Saúde, Curso de Farmácia, Universidade do Vale do Sapucaí - UNIVAS - \\ Pouso Alegre (MG) - Brasil. Tel: (35) 3449-2300 - E-mail: luajesica@yahoo.com.br \\ ${ }^{2}$ Fisioterapeuta, professor doutor. Instituto de Ciências Biológicas, Centro Universitário de Itajubá - FEPI - Itajubá \\ (MG) - Brasil. Av. Dr. Antônio Braga Filho, 687 - Varginha - Itajubá (MG). Tel: (35) 3629-8400 - E-mail: \\ lhfisio@yahoo.com.br \\ ${ }^{3}$ Bacharel em Educação Física. Professor mestre. Instituto de Ciências Biológicas, Centro Universitário de Itajubá - \\ FEPI - Itajubá (MG) - Brasil. Av. Dr. Antônio Braga Filho, 687 - Varginha - Itajubá (MG). Tel: (35) 3629-8400 - E- \\ mail: alexprofms@yahoo.com.br \\ ${ }^{4}$ Farmacêutico, professor mestre. Instituto de Ciências Biológicas, Curso de Farmácia, Centro Universitário de Itajubá - \\ FEPI - Itajubá (MG) - Brasil. Av. Dr. Antônio Braga Filho, 687 - Varginha - Itajubá (MG). Tel: (35) 3629-8400 - E- \\ mail: valdomirovagner@gmail.com \\ ${ }^{5}$ Farmacêutico, professor mestre. Faculdade de Enfermagem, Fundação Presidente Antônio Carlos de Itajubá - FUPAC \\ - Itajubá (MG) - Brasil. E-mail: valdomirovagner@gmail.com \\ ${ }^{6}$ Farmacêutico, professor mestre. Faculdade de Ciências da Saúde, Curso de Medicina, Universidade do Vale do \\ Sapucaí - UNIVAS - Pouso Alegre (MG) - Brasil. Tel: (35) 3449-2300 - E-mail: valdomirovagner@ gmail.com \\ * V.V. SOUZA. FEPI, Instituto de Ciências Biológicas, Faculdade de Farmácia, Centro Universitário de Itajubá - FEPI. \\ Av. Dr. Antônio Braga Filho, 687 - 37501-002 - Itajubá - Minas Gerais, Brasil. E-mail: valdomirovagner@gmail.com
}

Recebido em: 31/08/2013 - Aprovado em: 20/12/2013 - Disponibilizado em: 15/01/2014

RESUMO: A precariedade dos serviços de assistência à saúde e o baixo nível de conhecimento sobre medicamentos da população contrastam com a facilidade de se obter medicamentos sem receita médica, sendo isso, um incentivo para que a dispensação de medicamentos ocorra de forma desrespeitosa á legislação. O presente trabalho teve como objetivos principais analisar o nível de conhecimento da população a respeito de medicamentos ético, genérico e similar, bem como saber quais as principais fontes de informações a respeito. A pesquisa foi desenvolvida em três diferentes farmácias do Município de Silvianópolis, sendo aplicado aos pacientes um questionário visando a obtenção de informações. Dos 150 pacientes participantes da pesquisa, responderam corretamente as questões apenas: (15\%) questões sobre medicamentos de referência, (51\%) questões sobre medicamentos genéricos e (3\%) questões sobre medicamentos similares. A mídia sobrepujou os profissionais da saúde no que diz respeito à fonte de informação sobre medicamentos. De acordo com os resultados, pode-se concluir que os pacientes possuem escasso conhecimento a respeito de medicamentos de referência, genéricos e similares. Observou-se predomínio da visão mercantilista, tendo isso afetado a saúde da população negativamente.

Palavras-chave: Atenção Farmacêutica. Medicamentos. Uso racional. Reações adversas. Farmacoterapia 


\title{
EVALUATION OF LEVEL OF KNOWLEDGE AND QUALITY OF PHARMACEUTICAL CARE OFFERED A DRUG USERS IN SILVIANÓPOLIS, SOUTH OF MINAS, BRAZIL
}

\begin{abstract}
The precariousness of health care services and the low level of knowledge about medicines among contrast with the ease of obtaining drugs without a prescription, and therefore an incentive for dispensing drugs will occur disrespectfully legislation. This study aimed to analyze the main level of knowledge of the population about ethical drugs, generic and similar, as well as know what the main sources of information about it. The research was conducted in three different pharmacies Municipality Silvianópolis being applied to patients a questionnaire aimed at obtaining information. Of the 150 patients participating in the study, correctly answered questions only: (15\%) questions about drug reference $(51 \%)$ and questions about generic drugs (3\%) questions on similar drugs. The media overcame health professionals with regard to the source of drug information. According to the results, it can be concluded that patients who have little knowledge about the reference drugs, generic, and the like. Predominance of the mercantilist view, and it affected people's health negatively.
\end{abstract}

Keywords: Pharmaceutical Care. Medicines. Rational use. Adverse reactions. Pharmacotherapy

\section{INTRODUÇÃO}

O processo de educação em saúde tem como objetivo principal preparar os membros de uma comunidade para atuarem como um dos responsáveis na promoção da saúde, mediante interface entre saberes científicos, senso comum e as experiências vivenciadas, de maneira a promover continuamente a integração, a continuidade, a democratização do conhecimento e o avanço na área social. (ANGONESI, D. 2008)

Dentro o contexto atual, a educação em saúde consiste em um componente da promoção da saúde, tendo como meta a formação de indivíduos mais cientes dos fatores que podem ajudá-los a manter uma boa saúde e prevenir doenças. A informação sobre o uso adequado de medicamentos é parte relevante da educação em saúde, porém há muitas outras situações em que esse serviço pode ser prestado. (BONAL, J. 2008)
Tendo em vista tais evidências, é interessante que a educação em saúde envolva informações sobre as diferenças entre os medicamentos genéricos, éticos e similares, principalmente no que diz respeito a custobenefício. Sabe-se que os genéricos são medicamentos que possuem o mesmo princípio ativo, em igual dose e forma farmacêutica, comumente sendo administrados pela mesma via e possuindo, inclusive, a mesma indicação terapêutica do medicamento de referência, com o qual devem ser intercambiáveis. No entanto, as mesmas considerações não devem ser aplicadas aos medicamentos similares. A falta de informação quanto a estas diferenças pode inclusive, em alguns casos, afetar a farmacoterapia de forma negativa. (BARROS, J.C.A. 2000)

Este tipo de educação em saúde deve acontecer em farmácias e drogarias, devendo ser realizada principalmente por profissional 
farmacêutico e, este deve dispor de autonomia profissional para exercer tal função. $\mathrm{Na}$ prática, infelizmente, este tipo de educação em saúde não acontece de forma satisfatória, e seus serviços acabam sendo prejudicados por conflitos de interesse envolvendo profissionais não farmacêuticos. (FARINA \& ROMANO. 2009)

Portanto, os usuários de medicamentos possuem o direito da informação sobre os medicamentos de referência, genéricos e similares. Quesitos básicos como preço, equivalência, biodisponibilidade, seguimento do receituário, posologia, efeitos adversos e colaterais e o estímulo ao correto seguimento da farmacoterapia devem ser devidamente abordados pelo profissional farmacêutico no momento da dispensação, mediante embasamento científico e ético. (GALATO, D.; et al. 2008)

Os estudos até hoje desenvolvidos sobre medicamento genéricos envolvem principalmente a equivalência e biodisponibilidade dos fármacos em relação ao medicamento de referência, como também as dimensões de impacto que esses medicamentos têm causado no mercado farmacêutico. No entanto, existe uma lacuna em relação às implicações sociais que o medicamento genérico tem causado na população, principalmente em relação à confiabilidade e aceitabilidade desses produtos pela sociedade, mesmo o mesmo tendo vantagens consideráveis em relação aos outros citados. (MELO, D. O. et al. 2006)
Assim sendo, observa-se na prática que o uso do medicamento similar vem sendo estimulado nas farmácias, sem ao menos o paciente receber informações sobre as diferenças do mesmo em relação ao genérico e o referência. Ainda pior, muitas das vezes o paciente é induzido a optar pelo medicamento similar, não recebendo as devidas informações sobre a conseqüência desta substituição, tendo em vista que o medicamento similar nem sempre propicia níveis plasmáticos em velocidade e extensão adequados. (VIEIRA, F. S. \& ZUCCHI, P. 2006)

De mesmo modo, destaca-se que a população tem a opção de aceitar ou rejeitar as novas informações, podendo, todavia, adotar ou não novos comportamentos frente aos problemas de saúde. Assim, deve-se efetivar a educação em saúde num processo que estimule o diálogo, a indagação, a reflexão, o questionamento e a ação partilhada. (LUZ, T. C. B. et al. 2009)

Infelizmente existe um estímulo inadequado ao uso dos similares, que se deve pela visão meramente mercantilista dos donos de farmácia (não farmacêuticos), balconistas e técnicos de farmácias, que muitas das vezes induzem o paciente a optar pelos similares, mesmo sendo isso discrepante do que preconiza a legislação. Estes profissionais desconhecem os riscos que os medicamentos podem ocasionar a saúde dos pacientes, e não possuem os conhecimentos técnicos necessários para realização da dispensação. 
(LUZ, T. C. B. et al. 2009)

Além do mais, o paciente tem o direito de receber informações corretas, adequadas e de qualidade sobre os medicamentos a serem utilizados. O paciente tem o direito de avaliar o custo benefício do medicamento que esta adquirindo, sem ser induzido por falsas informações ou estímulos meramente mercantilistas. (LUZ, T. C. B. et al. 2009)

\section{MATERIAIS E MÉTODOS}

\section{Tipo e Área de estudo}

O trabalho realizado é do tipo descritivo transversal e de abordagem qualitativa. $\mathrm{O}$ estudo foi desenvolvido em três diferentes farmácias do Município de Silvianópolis. O quesito de escolha dos locais de estudo se baseou na preferência da população, isto é, selecionaram-se os locais mais acessíveis pela população do município.

\section{Amostra e sujeito da pesquisa}

Foram entrevistados 50 pacientes de cada estabelecimento, totalizando 150 indivíduos. A amostragem é do tipo probabilística aleatória simples, sendo incluídos todos os pacientes voluntários que aceitaram participar da pesquisa. Foram excluídos apenas os pacientes que, por ventura, decidiram não participar do estudo.

A coleta de dados foi realizada nos próprios estabelecimentos, após os pacientes
Diante disto, o propósito do presente estudo é avaliar a qualidade da educação em saúde ofertada nas farmácias e drogarias de um município, do Sul de Minas Gerais. Objetiva-se também a busca de alternativas que definitivamente melhorem a qualidade da Assistência e Atenção farmacêutica no município, visando principalmente o uso racional dos medicamentos.

serem atendidos pelos funcionários.

\section{Aspectos ético-legais da pesquisa}

Tendo em vista os aspectos legais exigidos pelo Ministério da Saúde para a realização de pesquisa com seres humanos, conforme a resolução 196/96 do Conselho Nacional de Saúde, a pesquisa somente foi iniciada após aprovação pelo Comitê de Ética e Pesquisa da Universidade do Vale do Sapucaí (UNIVAS-MG)

Após, forneceu-se aos pacientes as informações e os esclarecimentos necessários para a sua participação, demonstrando a relevância da pesquisa e sua importância no processo de aprendizagem acadêmica. Os mesmos foram informados sobre a liberdade de participar ou não, do sigilo das informações e do anonimato, sendo entregue a cada um dos participantes uma via do Termo de Livre Consentimento e Esclarecido.

\section{Coleta de dados}

Os dados dos sujeitos da pesquisa 
foram obtidos a partir de uma única fonte: os

dados fornecidos pelos mesmos durante as entrevistas.

\section{Dados dos pacientes}

Realizou-se a coleta de dados em uma única entrevista destinada a cada paciente com perguntas que investigaram qual o conhecimento da população a respeito de medicamentos de referência, genéricos e similares, buscando analisar também, qual a fonte de informação dos mesmos sobre os medicamentos.

O questionário foi aplicado aos pacientes dentro da própria farmácia, totalizando 10 entrevistas por dia, ocorrendo estas, diariamente, no período da manhã.

\section{Instrumentos para coleta de dados}

Foi utilizado para coleta de dados, um único questionário que continha uma forma direta e objetiva, constituído em sua maioria por questões não estruturadas.

\section{Análise dos dados}

Os dados colhidos foram tabulados em uma planilha, para apresentação de valores absolutos e percentuais, sendo que estes foram analisados individualmente $\mathrm{e}$ correlacionados.
Realizou-se também, uma análise comparativa com o total de entrevistados que souberam responder corretamente as questões e entrevistados que não souberam responder corretamente. As questões referentes às fontes de informação sobre medicamentos foram analisadas individualmente e as fontes de informações separadas. A análise foi dividida em duas categorias, sendo:

\section{(i) Nível de conhecimento e consumo da} população sobre medicamentos: para esta categoria foram inclusos itens destinados a verificar o conhecimento ou a falta de conhecimento da população em relação às três classes de medicamentos em estudo. Objetivou-se, desta forma, determinar a proporção de pacientes capazes de responder corretamente as questões do questionário, visando à análise do nível de conhecimento da população em relação ao assunto e, conseqüentemente, avaliar a qualidade da atenção farmacêutica fornecida aos pacientes.

\section{(ii) Fontes e avaliação da qualidade das} informações fornecidas à população: Nesta categoria foram incluídas questões relativas às fontes de informações dos pacientes sobre os medicamentos, visando descobrir de onde os pacientes adquirem informações sobre medicamentos e determinar quais as principais fontes de informações sobre medicamentos. 


\section{RESULTADOS E DISCUSSÃO}

Análise do nível do conhecimento dos pacientes

As características observadas nesta análise foram divididas em três classificações: conhecimento dos pacientes sobre medicamentos de referência, conhecimento dos pacientes sobre medicamentos genéricos, conhecimento dos pacientes sobre medicamentos similares. Os resultados sugerem que os pacientes praticamente desconhecem a diferença entre as três classes de medicamentos, não sabendo em alguns casos, inclusive, que os genéricos possuem preço mais acessível com a mesma qualidade e segurança dos medicamentos de referência. A seguir, os resultados em detalhes.

\section{Conhecimento dos pacientes sobre medicamentos de referência}

Apenas $15 \%$ souberam responder corretamente a esta questão, sendo que, $85 \%$ dos pacientes analisados forneceram respostas completamente incorretas (FIGURA 1).
Figura 1 - Distribuição dos pacientes de acordo com o nível de conhecimento sobre medicamentos de referência

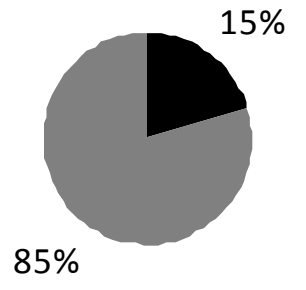

Pessoas que souberam responder Pessoas que não souberam responder

Fonte - Dos próprios autores.

\section{Conhecimento dos pacientes sobre medicamentos genéricos}

Como se pode observar na FIGURA 2, apenas $51 \%$ soube responder corretamente a esta questão.

Figura 2. Distribuição dos pacientes de acordo com o nível de conhecimento sobre medicamentos genéricos.

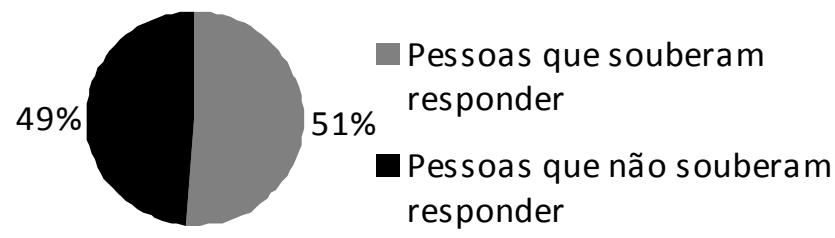

Fonte - Dos próprios autores. 


\section{Conhecimento dos pacientes sobre medicamentos similares}

Apenas 23\% da amostra analisada souberam responder corretamente. (FIGURA $3)$.

Figura 3: Distribuição dos pacientes de acordo com o nível de conhecimento sobre medicamentos similares.

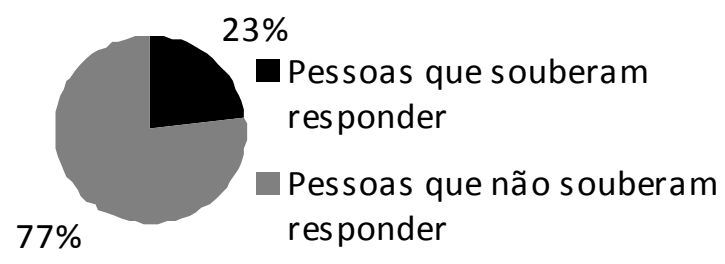

Fonte - Dos próprios autores.

\section{Fontes de informações dos pacientes}

Para avaliação deste item, cada paciente poderia responder a questão citando uma ou mais fontes de informações. Apenas $15 \%$ dos pacientes relataram ter obtido informações em farmácias. (Figura 4)
Figura 4. Principais fontes de informações sobre medicamentos.

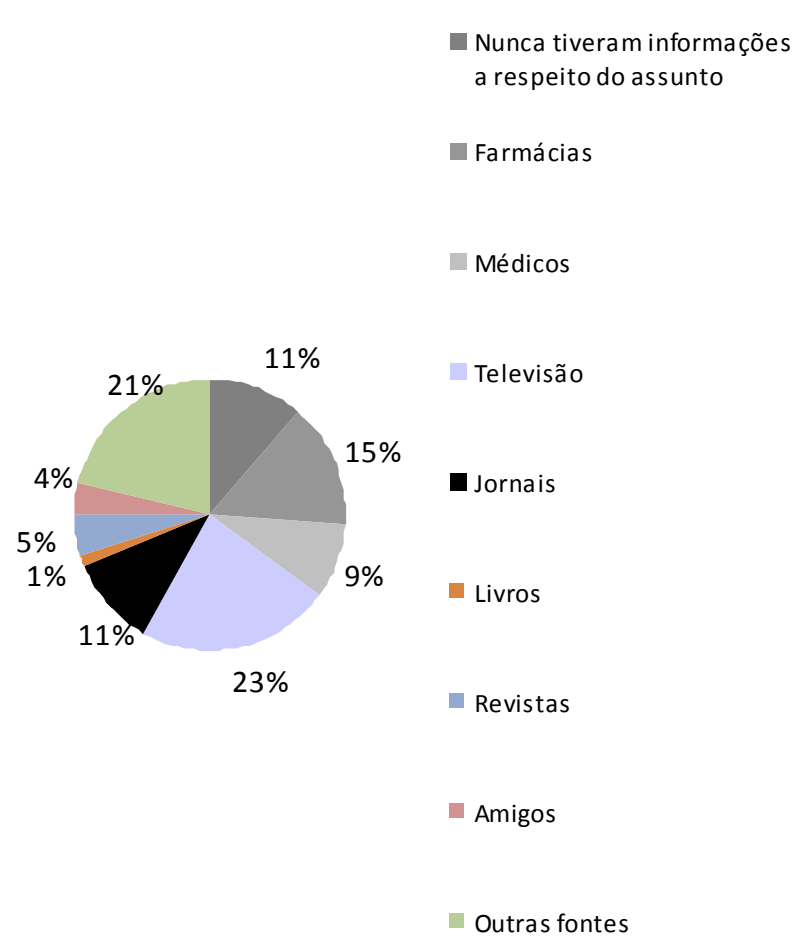

Fonte - Dos próprios autores.

Com base nos resultados, alguns pontos merecem destaque especial. A seguir, proporse-á breve discussão sobre tais itens. Todavia, diversos estudos, como o de (SOUZA. 2008) confirmam a necessidade da oferta da AFD para a garantia da saúde da população. Contudo, pouco se avançou em Atenção Farmacêutica, no Brasil, desde sua proposta em 1990 por (HEPLER. 1990)

De acordo com (ROCHA, et al. 2007), vale salientar que os indivíduos desprovidos de conhecimento apresentam dificuldades de entendimento quanto à correta e racional utilização dos medicamentos, da orientação 
prestada pelo profissional de saúde e da compreensão sobre o seu estado de saúde. Ressaltemos, ademais, que a troca de informações entre o prescritor e o paciente determinará a indicação do medicamento a ser prescrito.

Neste ciclo não se pode descartar a contribuição do farmacêutico como ator que provê informações sobre o uso do medicamento - possíveis interações, efeitos adversos, efeito terapêutico, dose do medicamento, posologia, cuidados especiais no armazenamento.

Ademais, o acúmulo destes conhecimentos pelo paciente favorece a assimilação de informações a respeito do produto - efeito terapêutico, contraindicações, efeitos colaterais. Adicionalmente, entre outros aspectos, o grau de conhecimento melhora a interação médico-pacientefarmacêutico.

Quando se questionou os pacientes quanto a medicamentos de referência, algumas respostas merecem destaque por ressaltarem a necessidade imediata de melhoria do nível de educação em saúde oferecidos aos pacientes: "Para mim são alguma coisa de comida"; "são os controlados"; "são os medicamentos utilizados para combater doenças graves como o câncer e a AIDS"; "são os analgésicos"; "são os medicamentos de uso contínuo", "são os medicamentos indicados por alguma pessoa e comprados sem receita médica”.
Quando os sujeitos da pesquisa foram interrogados a respeito de medicamentos genéricos, algumas afirmativas sugerem possíveis conflitos de interesse por parte de alguns profissionais da saúde, por exemplo: "são medicamentos falsos, não confiáveis"; "são os medicamentos originais"; "os médicos recomendam que não sejam usados medicamentos genéricos e sim os de marca”.

De mesmo modo, quando questionados sobre medicamentos similares, surgiram respostas que também sugerem conflitos de interesse e mercantilismo por parte de alguns profissionais: "são medicamentos a base de farinha; "o similar é uma cópia do original, é o mesmo que genérico"; "os medicamentos similares são iguais aos outros"; “os similares são melhores que os genéricos e mais baratos".

Outro resultado que merece destaque é a desprezível participação das farmácias no que diz respeito à orientação e educação em saúde. Observou-se que a mídia (rádio, TV, etc) sobrepujam as fontes de informações confiáveis (médicos, farmacêuticos, etc).

Neste sentido, tais resultados são condizentes com os observados por (LAGE et al. 2005). Neste estudo, ficou evidenciado que a informação e a propaganda promocional de medicamentos podem influenciar em grande medida a forma pela qual eles são utilizados. Tais estudos apontam que as informações sobre medicamentos veiculadas na mídia são tendenciosas, predominando as boas notícias e a supervalorização das propriedades 
medicamentosas.

Em concordância, (BARROS. 2000) também sugere que informação disponível sobre os medicamentos, destinada ou não aos prescritores, é produzida e disseminada, em grande medida, pelos próprios fabricantes. Visando objetivos mais comerciais que sanitários, é claro o caráter tendencioso desse tipo de informação.

\section{CONCLUSÃO}

De acordo com os resultados, pode-se concluir que os pacientes possuem escasso conhecimento a respeito de medicamentos de referência, genéricos e similares.

Observou-se que a visão mercantilista tem predominado. Neste sentido, a saúde da população tem sido afetada negativamente não somente pela automedicação, mas também

\section{REFERÊNCIAS}

ANGONESI, D. Dispensação farmacêutica: uma análise de diferentes conceitos e modelos. Rev. Ci. da saúde coletiva, Rio de Janeiro, v.13, n.1, p. 629-640, 2008.

BARROS, J.C.A. Informação sobre medicamentos: o duplo padrão de conduta das empresas farmacêuticas. Cad. de Saúde Pública, Rio de Janeiro, v.16, n.2, p.427, 2000.

BONAL, J. Experiências em Atenção Farmacêutica. In: STORPIRTIS, S.; MORI, A. L. P. M.; YOCHIY, A.; RIBEIRO, E.; PORTA, V. (Eds.). Ci.s Farm-s. Farm. Clín. e Atenção Farmacêutica. São Paulo: Guanabara Koogan, 2008. cap.44, p.451-460. pelo uso irracional e desnecessário de medicamentos, fruto do incentivo que a mídia oferece.

Em conformidade com os dados obtidos durante a pesquisa pode-se notar que a qualidade da atenção farmacêutica oferecida no município é precária ou praticamente inexistente. Acredita-se que tal observação condiz com a realidade de muitos municípios do país. Há, portanto, a necessidade de promover uma conscientização dos profissionais farmacêuticos para que, de fato, ofertem a Atenção Farmacêutica.

Neste sentido, acredita-se que somente mudanças na legislação poderiam minimizar essa problemática. Estudos mais específicos se fazem necessários.

FARINA, S.S.; ROMANO, N.S. Atenção farmacêutica em farmácias e drogarias: existe um processo de mudança? Rev. Saúde e Sociedade, v.18, n.1, p.7-18, 2009.

GALATO, D.; et al. A dispensação de medicamentos: uma reflexão sobre o processo para prevenção, identificação e resolução de problemas relacionados à farmacoterapia. Rev. Bras. de Ci-s Farmacêuticas, São Paulo, v.44, n.3, p. 465-475, 2008.

HEPLER, C. D. STRAND, L. M. Opportunities and responsibilities in the pharmaceutical care. Am. J. Hosp. Pharm., v.47, n.3, p.533-543. 1990. 
LUZ, T. C. B.; LOYOLA F.; LIMA, C.A.I.; et $a l$. Estudo de base populacional da subutilização de medicamentos por motivos financeiros entre idosos na Região Metropolitana de Belo Horizonte. Cad. de Saúde Pública, Rio de Janeiro, v.25, n.7, p. 1578-1586, 2009.

MELO, D. O.; RIBEIRO, E; STORPIRTIS, S. A importância e a história dos estudos de utilização de medicamentos. Rev. Bras. de Cis Farmacêuticas, São Paulo, v.42, n.4, p. 475485, 2006.

LAGE, E.A.; FREITAS, M.I.F.; ACURCIO, F.A. Informação sobre medicamentos na imprensa: uma contribuição para o uso racional? Ci. saúde coletiva, Rio de Janeiro, v. 10, n. 1, p. 133-139, 2010.
ROCHA, C. E.; BARROS, J.A.C.; SILVA, M.D.P. Levantamento de dados sobre o conhecimento e informação acerca dos medicamentos genéricos em uma população de pacientes do serviço de saúde ambulatorial do Recife, Pernambuco, Brasil. Cad. de Saúde Pública, São Paulo, v.23, n.5, p.1141, 2007.

SOUZA, V. V.; BERTONCIN, A.L.F. Atenção Farmacêutica para pacientes hipertensos Nova metodologia e a importância dessa prática no acompanhamento domiciliar. Rev. Bras. de Prom. a Saúde, Fortaleza, v.21, n.3, p. 224-230, 2008.

VIEIRA, F. S.; ZUCCHI, P. Diferenças de preços entre medicamentos genéricos e de referência no Brasil. Rev. de Saúde Pública, São Paulo, v.40, n.3, p.444-449, 2006. 